\title{
FAST TRANSVERSE INSTABILITY IN THE NSNS ACCUMULATOR RING
}

\author{
Alessandro G. Ruggiero and Mike Blaskiewicz \\ AGS Department, Brookhaven National Laboratory, Upton, NY 11973.
}

\section{Abstract*}

This paper reports on the results of investigation of possible fast transverse instabilities in the NSNS Accumulator Ring. The instability may be caused by the presence of stripline devices like kicker magnets, the active damper system, and by the RF cavities, and the sharp steps of the vacuum pipe. The instability can be overcome by adopting aluminum as the material of the vacuum chamber, and by smoothing the shape of the vacuum pipe. Still the growth time of the instability remains short especially for the mode in proximity of the betatron tune.

\section{THE NSNS ACCUMULATOR RING}

The function of the Accumulator Ring is to take the 1.0 $\mathrm{GeV}$ proton beam from the Linac and convert the long Linac beam pulse of about $1 \mathrm{~ms}$ into a 0.5 microsecond beam in about 1100 turns. The bunch compression occurs during the injection process, and the beam is immediately extracted at the end of the process. The final beam has an intensity of $2.08 \times 10^{14}$ protons per pulse, resulting in 2 MW average beam power at $60 \mathrm{~Hz}$ repetition rate. The lattice of the Accumulator Ring is a simple FODO lattice with four-fold symmetry [1]. The salient design parameters are shown in Table 1.

\section{THE RING VACUUM PIPE}

The main parameters that enter the investigation of the coherent transverse instability are the dimension and the shape of the vacuum chamber, and the resistivity of the wall. The NSNS Accumulator Ring is made of three sections with different vacuum chamber shape and size. The analysis that was made uses formulae that apply strictly to circular geometry. Thus we have approximated the rectangular vacuum chamber by a circular one, and, by taking an average of the pipe dimension around the ring, we have assumed a pipe with an internal radius $b=10 \mathrm{~cm}$. The material of the vacuum chamber is stainless steel for $15 \%$ of the circumference, and aluminum for the rest.

\section{TRANSVERSE BEAM DIMENSION}

The betatron emittance has the same value in the two planes of oscillation. We adopted the criterion to define the total emittance $\varepsilon_{\text {tot }}$ as 5 times the rms emittance $\varepsilon_{\text {rms }}$.

* Research on the NSNS is sponsored by the Division of Material Sciences, U.S. Department of Energy, under contract number DE-AC05-96OR22464 with Lockheed Martin Energy Research Corporation for Oak Ridge National Laboratory.
Define the average values of the envelope functions $\left\langle\beta_{\mathrm{H}, \mathrm{V}}\right\rangle=\mathrm{R} / \mathrm{Q}_{\mathrm{H}, \mathrm{V}}$, with $\mathrm{R}$ the average closed orbit radius and $\mathrm{Q}_{\mathrm{H}, \mathrm{V}}$ the betatron tunes. The average rms beam size in the vertical and horizontal plane are given by $\sigma_{\mathrm{H}, \mathrm{V}}{ }^{2}=$ $\left\langle\beta_{\mathrm{H}, \mathrm{V}}\right\rangle \varepsilon_{\mathrm{rms}}$. This is the contribution from the betatron motion alone, to which we should add the contribution in the horizontal plane from the relative momentum spread $\delta$ in the beam, which is $\sigma_{\mathrm{E}}=\langle\eta\rangle \delta$, where $\langle\eta\rangle=\mathrm{R} / \gamma_{\mathrm{T}}{ }^{2}$ is the average value of the dispersion around the ring. The total beam relative momentum spread $\Delta$ is given in Table 1. The rms value $\delta$ is $1 / 5$ of the full value.

Table 1: Parameters of the NSNS Accumulator Ring

\begin{tabular}{|l|r|}
\hline Average Power & $2 \mathrm{MW}$ \\
\hline Kinetic Energy & $1.0 \mathrm{GeV}$ \\
\hline Circumference, $2 \pi \mathrm{R}$ & $220.7 \mathrm{~m}$ \\
\hline Number of Protons, $\mathrm{N}$ & $2.08 \times 10^{14}$ \\
\hline Betatron Tunes, $\mathrm{Q}_{\mathrm{H} / \mathrm{V}}$ & $5.82 / 5.82$ \\
\hline Transition Energy, $\gamma_{\mathrm{T}}$ & 4.93 \\
\hline Full Betatron Emittance, $\varepsilon_{\mathrm{tot}}$ & $<0.2$ \\
\hline Space-Charge Tune-Shift & $10 \mathrm{eV}-\mathrm{s}$ \\
\hline Total Bunch Area, $\mathrm{S}$ & $546.6 \mathrm{~ns}$ \\
\hline Full Bunch Length, L & $1.6 \%$ \\
\hline Full Momentum Spread, $\Delta$ & $3.80 \mathrm{~cm}$ \\
\hline Average Beam radius, a & $10 \mathrm{~cm}$ \\
\hline Average Pipe Radius, b & $\pi 00$ \\
\hline
\end{tabular}

\section{LONGITUDINAL BEAM DIMENSION}

The total bunch area is $\mathrm{S}=10 \mathrm{eV}-\mathrm{s}$. We shall assume a single RF cavity system for the bunch compression. The requirement is a beam gap, free of any particles, of 294.7 ns and a full bunch length $\mathrm{L}=546.6 \mathrm{~ns}$. Assuming again, as usual, a ratio of factor 5 between total and rms values, we can estimate the rms bunch length $\sigma$ and rms momentum spread $\delta$. The RF system operates at the harmonic number $\mathrm{h}=1$, and there is thus a single beam bunch, which we assume to hold a cos-square distribution. 


\section{THE FAST TRANSVERSE INSTABILITY}

The synchrotron period is $0.9 \mathrm{~ms}$, so that the beam bunch will complete only one full synchrotron oscillation before it is extracted. The growth rate of any transverse instability, in order to have a consequence to the beam disruption and loss, will have to be considerably larger than the synchrotron frequency. This situation is characterized as a fast transverse instability, where the synchrotron motion can be neglected and the beam bunch can be treated as a chopped section of coasting beam. The wellknown coasting beam theory [2,3] can then be applied.

A coherent perturbation may develop around the contour of the beam bunch. The wavelength of this perturbation has to be at least the bunch length. This sets a lower limit to the perturbation frequency. In terms of the revolution harmonic number this limit is $n>5$. The upper limit is set by the cut-off due to the presence of the vacuum chamber which is given by $n<\gamma R / b \sim 726$. Outside this range a transverse coherent instability cannot be expected.

\section{THE THEORY OF TRANSVERSE COHERENT INSTABILITIES FOR COASTING BEAMS}

The stability condition, in terms of the transverse coupling impedance $\mathrm{Z}_{\mathrm{T}}$ is

$$
\begin{aligned}
\left|\mathrm{Z}_{\mathrm{T}}\right|< & \mathrm{E}_{0} \pi \mathrm{Q}_{\mathrm{H}, \mathrm{V}} \beta \gamma\left[\left|\left(\mathrm{n}-\mathrm{Q}_{\mathrm{H}, \mathrm{V}}\right) \eta+\xi_{\mathrm{H}, \mathrm{V}}\right|(\Delta \mathrm{p} / \mathrm{p})\right. \\
& +\delta \mathrm{v}] / \mathrm{e} \mathrm{I}_{\mathrm{p}} \mathrm{R}=\mathrm{Z}_{\text {beam }}
\end{aligned}
$$

where $\Delta \mathrm{p} / \mathrm{p}$ is the FWHM value of the beam momentum spread, $\mathrm{E}_{0}$ is the proton rest energy, $\xi_{\mathrm{H}, \mathrm{V}}$ the chromaticity, and $\delta v$ the betatron tune spread from non linear elements like octupole magnets. $\mathrm{I}_{\mathrm{p}}$ is the peak current of the bunch

$$
\mathrm{I}_{\mathrm{p}}=\mathrm{Ne} /(2 \pi)^{1 / 2} \sigma \text {. }
$$

In absence of Landau damping, one can estimate the growth rate of the coherent instability

$$
\tau^{-1}=\mathrm{I}_{\mathrm{p}} \mathrm{r}_{\mathrm{p}} \operatorname{Re}\left(\mathrm{Z}_{\mathrm{T}}\right) / \mathrm{e} \mathrm{Q}_{\mathrm{H}, \mathrm{V}} \gamma \mathrm{Z}_{0}
$$

where $\mathrm{Z}_{0}=377 \mathrm{ohm}$, and $\mathrm{r}_{\mathrm{p}}=1.535 \times 10^{-18} \mathrm{~m}$.

As we shall see below, we are in a case where the space charge contribution to the coupling impedance dominates. The frequency of the coherent instability suffers a large shift. This is an indication that there is no Landau damping naturally built within the beam large enough to compensate for the shift, unless this is introduced externally. The real frequency shift of the coherent instability is given by

$$
\Delta \omega=\mathrm{I}_{\mathrm{p}} \mathrm{r}_{\mathrm{p}} \operatorname{Im}\left(\mathrm{Z}_{\mathrm{T}}\right) / \mathrm{e} \mathrm{Q}_{\mathrm{H}, \mathrm{V}} \gamma \mathrm{Z}_{0}
$$

\section{THE TRANSVERSE COUPLING IMPEDANCE}

There are four major contributions [4] to the transverse coupling impedance. The space-charge contribution domi- nates in a low-energy storage ring:

$$
Z_{T}=i R Z_{0}\left(a^{-2}-b^{-2}\right) / \beta^{2} \gamma^{2} .
$$

Next, we have the contribution from the wall resistivity:

$\mathrm{Z}_{\mathrm{T}}=(1-\mathrm{i}) \mathrm{R}\left[2 \mathrm{R} \mathrm{Z}_{0} \rho_{\mathrm{W}} / \beta\left(\mathrm{n}-\mathrm{Q}_{\mathrm{H}, \mathrm{V}}\right)\right]^{1 / 2} / \mathrm{b}^{3}$.

By virtue of the deflection theorem [4], the longitudinal coupling impedance $\mathrm{Z} / \mathrm{n}$ can be translated into an equivalent transverse coupling impedance:

$$
\mathrm{Z}_{\mathrm{T}}=2 \mathrm{RZ} / \beta \mathrm{b}^{2}\left(\mathrm{n}-\mathrm{Q}_{\mathrm{H}, \mathrm{V}}\right) .
$$

The wall components included in the deflection mode analysis are: bellows, striplines for the beam position monitors and the active damper system, kicker magnets, vacuum chamber steps, vacuum ports, and RF cavities [5].

A large contribution to the real part of the impedance comes from the vacuum chamber steps. The longitudinal coupling impedance from $\mathrm{M}$ single steps (uncoupled) was estimated originally by $\mathrm{H}$. Hereward [6]

$$
\begin{aligned}
\mathrm{Z} / \mathrm{n} & =2 \mathrm{M}(1-\mathrm{i} \pi) \mathrm{Z}_{0}(\mathrm{~W}-1)^{2} \mathrm{~b} / 2 \pi^{2} \mathrm{R} \\
\text { for } \mathrm{n}<\mathrm{n}_{\mathrm{W}} & =2 \pi \mathrm{R} / 2 \mathrm{~b}(\mathrm{~W}-1), \text { and for } \mathrm{n}>\mathrm{n}_{\mathrm{W}} \\
\mathrm{Z} / \mathrm{n} & =\mathrm{Z}_{0} \mathrm{M}(\mathrm{W}-1) / 2 \pi \mathrm{n}
\end{aligned}
$$

where $\mathrm{W}=\mathrm{b}_{2} / \mathrm{b}_{1}$ is the ratio of the outer dimension $\mathrm{b}_{2}$ to the inner dimension $b_{1}$ of the step.

According to this formula a substantial resistive contribution occurs in the low frequency range. One should point out that very likely this result really applies only to wavelengths which have about the pipe dimension, that is in the proximity of the cut-off. Moreover, steps come in pairs, an entrance followed by an exit discontinuity. Thus, one deals in reality with resonating cavities rather than single de-coupled steps. The contribution represented by Eq.s ( 8 and 9) has been originally included in the analysis also for very low frequencies, but we believe that otherwise it should really be neglected.

Though it was clearly demonstrated that relation (7) between longitudinal and transverse coupling impedance holds well for stripline devices, like beam position monitors, kickers and the damper system [7], there are some questions about its validity for cavity-like objects, like bellows, vacuum chamber steps, vacuum ports, and RF cavities. Nonetheless, also for these components we have calculated the longitudinal coupling impedance [5] and have then derived the transverse coupling impedance in combination with Eq.(7).

\section{THE NSNS ACCUMULATOR RING}

Figure 1 is the display of the total expected transverse coupling impedance versus the harmonic number $n$ for the 
NSNS Accumulator Ring. The peak around $n=6$ is caused by the dependence with the betatron tunes as shown both in Eq.s (6 and 7).

To determine the stability of the beam, we calculate the difference $\left|Z_{T}\right|-Z_{\text {beam }}$, taking $\delta v=0$. It is seen that the beam is unstable for all modes $n<100$. The beam is stable for large mode numbers as a consequence of the frequency compaction factor $\eta$.

The growth time $\tau$ of the instability, in absence of Landau damping, is plotted in Figure 2. The minimum is $\tau=$ $5.3 \mu$ s for $\mathrm{n}=6$. At $\mathrm{n}=7$ the growth time $\tau=40 \mu \mathrm{s}$, and at $\mathrm{n}=8, \tau=91 \mu \mathrm{s}$.

Finally, Figure 3 is the plot of the real frequency shift $\Delta \omega$, divided by the angular revolution frequency $2 \pi f_{0}$. Since the shift is only a small fraction of one harmonic, the possibility of overlapping of neighboring stopbands (mode coupling) seems to be excluded. Moreover, $\Delta \omega / 2 \pi f_{0}$ is about the amount of tune spread which is required to make the motion stable.

\section{HOW TO REDUCE THE GROWTH RATE OF THE INSTABILITY}

The major concern is obviously the instability for those modes in proximity of the betatron tune. The fewer modes below $n=6$ are not relevant in this analysis, since they cannot be excited by the beam. The worst case is represented by the mode $n=6$. All the modes $n>20$ have growth times of at least half a millisecond, and thus have no significant consequences to the beam performance. The imaginary part of the transverse coupling impedance is essentially given by the space-charge term. The contribution from the other components are entirely negligible.

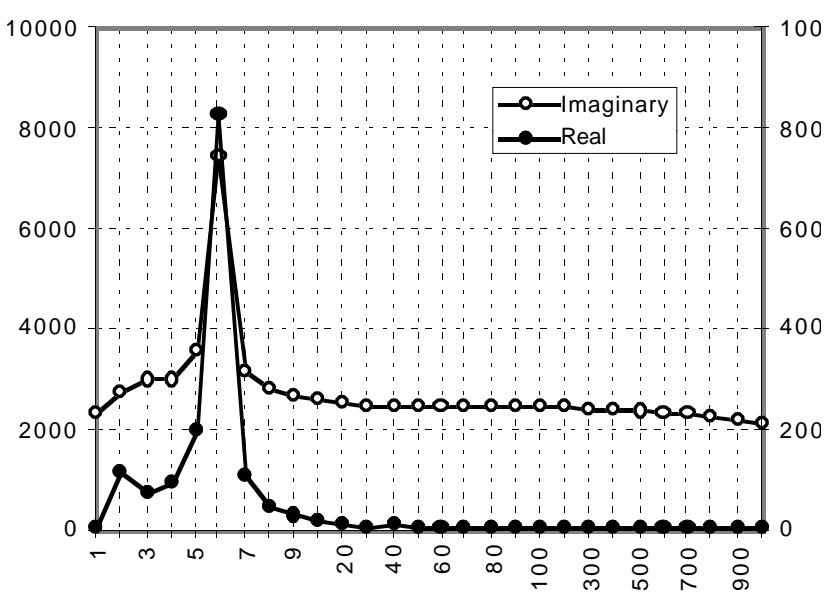

Figure 1. The Transverse Coupling Impedance $\mathrm{Z}_{\mathrm{T}}$ (in kohm/m) vs. the Harmonic Number n.

But the largest contribution to the resistive part of the impedance at $n=6$ comes (in the order) from the kicker magnets (409 kohm/m), the vacuum chamber steps (168 $\mathrm{kohm} / \mathrm{m}$ ), the RF cavities (147 kohm/m), and the striplines of the active damper system $(103 \mathrm{kohm} / \mathrm{m})$. The contribu- tion from the wall resistivity is very small $(2.8 \mathrm{kohm} / \mathrm{m})$ when compared to the other components. At the same time the resistive contribution of the beam position monitor is only $2.7 \mathrm{kohm} / \mathrm{m}$

Lowering the betatron tune from 5.8 to 5.2 causes an increase of the instability grow time by a factor of four. At $\mathrm{n}=6$ now $\tau=21 \mu \mathrm{s}$. Though this is a significant improvement, nevertheless clearly is not enough. We have pointed out that there are four major components which need very careful engineering design to lower their resistive contribution.

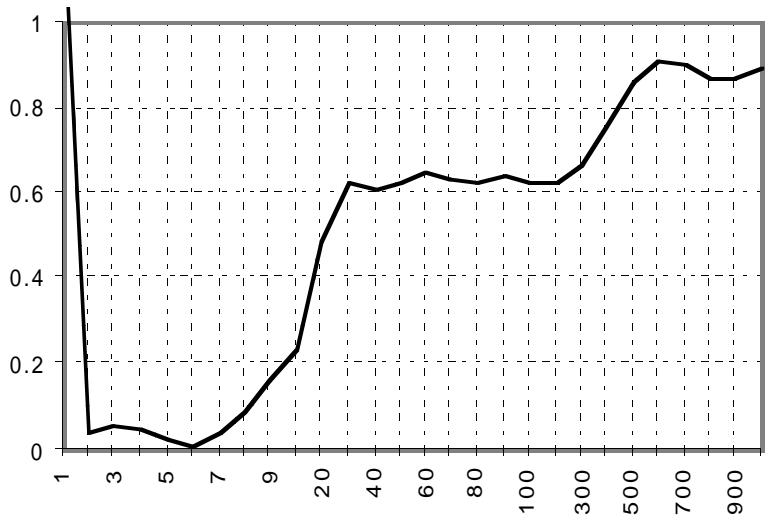

Figure 2. Growth time $\tau$ of the Instability (in ms).

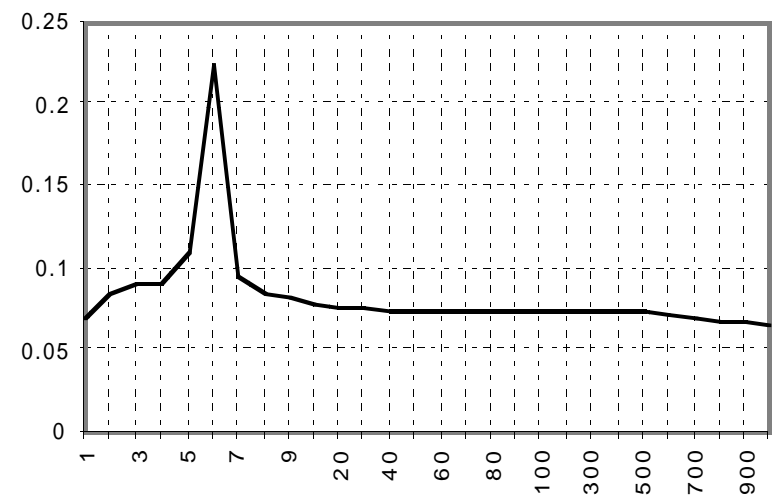

Figure 3. The Real Frequency-Shift $\Delta \omega / 2 \pi f_{0}$.

\section{REFERENCES}

[1] W.T. Weng et al. These Proceedings.

[2] L.J. Laslett, V.K. Neil, A.M. Sessler, Rev. Sci. Instr., 36, 436, (1965).

[3] K. Hubner, A.G. Ruggiero, V.G. Vaccaro, Proc.7th Int. Conf. on High Energy Accel., Yerevan, p. 343, (1969)

[4] F. Sacherer, Proc. of 9th Int. Conf. on High Energy Accele., SLAC, p. 347, (May 1974).

[5] A.G. Ruggiero, BNL/NSNS Technical Notes No. 27 and 28. April, 1997.

[6] H. Hereward, CERN / ISR - DI / 75-47 (Oct. 1975).

[7] A.G. Ruggiero and V.G. Vaccaro, Internal Report LNF-69/70, Frascati (Italy), Nov. 1969. 